\title{
EDITORIAL
}

\section{Kidney disease in lupus is not always 'lupus nephritis'}

\author{
Hans-Joachim Anders*1 and Jan J Weening ${ }^{2,3}$ \\ See related research by Song et al., http://arthritis-research.com/content/15/1/R12
}

\begin{abstract}
In lupus erythematosus, elevated serum creatinine levels and urinary abnormalities implicate a kidney disorder, which may not always be lupus nephritis as defined by the current classification of the International Society of Nephrology/Renal Pathology Society. The signs of renal dysfunction may be caused by lupusunrelated renal injury such as drug toxicity or infection or by lupus-associated mechanisms that are not part of the classification, such as minimal change nephrotic syndrome or thrombotic microangiopathy. The latter seems to complicate lupus nephritis more frequently than previously thought. An unbiased assessment of kidney disease in lupus requires a kidney (re-)biopsy to define the appropriate management.
\end{abstract}

In the previous issue of Arthritis Research \& Therapy, Song and colleagues [1] discussed specific kidney abnormalities in patients with lupus erythematosus (LE). LE can manifest as cutaneous LE or as a systemic disorder (systemic lupus erythematosus, or SLE) [2]. In patients with LE, renal abnormalities, such as elevated serum creatinine levels or urinary abnormalities, require further diagnostic work-up because they may indicate renal involvement in SLE or an unrelated form of renal disease [3]. Furthermore, renal symptoms may indicate flares of disease activity in patients with established lupus nephritis but may also reflect renal injury due to concomitant toxicity or infectious disease. How should lupus nephritis be defined? The current classification of the International Society of Nephrology/Renal Pathology Society (ISN/RPS) is based on the phenotype of immune complex glomerulonephritis that depends on the predominant site of immune complex deposition within the glomerulus and on different stages of acute (and

*Correspondence: hjanders@med.uni-muenchen.de

'Renal Division, Medizinische Klinik und Poliklinik IV, Klinikum der Universität

München, Ziemssenstraße 1, 80336 Munich, Germany

Full list of author information is available at the end of the article potentially reversible) injury and chronic (and potentially irreversible) scarring [4]. The article on ISN/RPS classification of lupus nephritis [4] emphasizes the importance of including all other forms of kidney injury, separate from immune complex-mediated glomerulonephritis, in the diagnostic evaluation of the renal biopsy in SLE, including thrombotic microangiopathy (TMA). The article advocates that 'the extent, severity, and type of tubulointerstitial injury (tubular atrophy, interstitial fibrosis, and chronic lesions) and vascular disease (vascular deposits, thrombi, vasculitis, sclerosis) should also be documented and graded (mild, moderate, severe) in the diagnostic line' [4].

Song and colleagues [1] presented a detailed histopathological analysis of a series of 148 patients with biopsy-proven lupus nephritis, focusing on the prevalence of TMA. The authors defined TMA by light microscopy as interlobular artery, arteriole, and glomerular capillary lesions, including endothelial swelling, lumen narrowing or obliteration, and thrombi formation. Electron microscopic TMA criteria are swelling of glomerular endothelial cells, detachment from glomerular basement membrane, and widening of the subendothelial space. Fibrin staining identifies acute microthrombi, whereas mucoid changes and onionskin lesions of arterioles were considered chronic. The authors found such TMA lesions in 36 out of the 148 patients with lupus nephritis [1]. In some of these cases, TMA was a manifestation of associated systemic autoimmune states such as the anti-phospholipid antibody syndrome, scleroderma, or thrombotic thrombocytopenic purpura, which have specific diagnostic criteria and are not included as a specific category in the current ISN/RPS classification of lupus nephritis [4].

In the majority of cases, TMA was associated with immune complex-mediated forms of lupus nephritis. According to the authors, such cases can be missed easily unless fibrin immunostaining is performed. TMA results from severe injury to the vascular endothelium, which Song and colleagues [1] found to be associated with poorer renal outcomes as compared with patients with non-TMA lupus nephritis. As such, reporting TMA in the renal biopsy and defining the ISN/RPS class are important, even though the management implications 
Table 1. Types of kidney disease in patients with systemic lupus erythematosus

Immune complex glomerulonephritis ('lupus nephritis')

Immune complex tubulointersitial nephritis

Minimal change nephrotic syndrome

Thrombotic microangiopathy

Infectious ascending tubulointerstitial disease

Opportunistic renal infections

Renal drug-induced toxicity

Renal injury due to concomitant disease (for example, hypertension and diabetes mellitus)

Amyloidosis

remain unclear unless a causative disorder such as the anti-phospholipid antibody syndrome (anticoagulation), overlap syndrome with scleroderma (angiotensin inhibition), or thrombotic thrombocytopenic purpura (plasma exchange) can be identified.

The report by Song and colleagues reminds us that, in LE, elevated serum creatinine levels or urinary abnormalities (or both) may not always represent immune complex glomerulonephritis (that is, 'lupus nephritis'). In addition, patients with SLE can develop other kidney disorders that may be related or unrelated to SLE and SLE management (Table 1) [3].

\section{Abbreviations}

ISN/RPS, International Society of Nephrology/Renal Pathology Society; LE, lupus erythematosus; SLE, systemic lupus erythematosus; TMA, thrombotic microangiopathy.

\section{Competing interests}

The authors declare that they have no competing interests.

\section{Author details}

${ }^{1}$ Renal Division, Medizinische Klinik und Poliklinik IV, Klinikum der Universität München, Ziemssenstraße 1, 80336 Munich, Germany. ${ }^{2}$ Department of Pathology, Erasmus University Medical Center, Postbus 2040, 3000 Rotterdam, The Netherlands. ${ }^{3}$ Department of Pathology, Tergooiziekenhuizen, Postbus 10016, 1201 Hilversum, The Netherlands.

Published: 1 March 2013

\section{References}

1. Song D, Wu LH, Wang FM, Yang XW, Zhu D, Chen M, Yu F, Liu G, Zhao MH: The spectrum of thrombotic microangiopathy in lupus nephritis. Arthritis Res Ther 2013, 15:R12.

2. Rahman A, Isenberg DA: Systemic lupus erythematosus. N Engl J Med 2008, 358:929-939.

3. Anders HJ, Vielhauer V: Renal co-morbidity in patients with rheumatic diseases. Arthritis Res Ther 2011, 13:222.

4. Weening JJ, D'Agati VD, Schwartz MM, Seshan SV, Alpers CE, Appel GB, Balow JE, Bruijn JA, Cook T, Ferrario F, Fogo AB, Ginzler EM, Hebert L, Hill G, Hill P, Jennette JC, Kong NC, Lesavre P, Lockshin M, Looi LM, Makino H, Moura LA, Nagata M: The classification of glomerulonephritis in systemic lupus erythematosus revisited. J Am Soc Nephrol 2004, 15:241-250.

doi:10.1186/ar4166

Cite this article as: Anders $\mathrm{HJ}$, Weening JJ: Kidney disease in lupus is not always 'lupus nephritis'. Arthritis Research \& Therapy 2013, 15:108. 